\title{
Effect of Dipeptidyl Peptidase- 4 (DPP-4) Inhibitor on Biomarkers of Kidney Injury and Vascular Calcification in Diabetic Nephropathy: A Randomized Controlled Trial
}

Thananda Trakarnvanich ( $\square$ thananda@hotmail.com )

Navamindradhiraj University

\section{Bancha Satirapoj}

Division of Nephrology, Department of Medicine Pramongkutklao Hospital

\section{Sawangjit Suraamornkul}

Navamindradhiraj University

\section{Thanit Chiranantawat}

Department of Medicine, Police General Hospital

\section{Anoma Sanpatchayapong}

Navamindradhiraj University

\section{Torpong Chaimon}

Navamindradhiraj University

\section{Research Article}

Keywords: Gemigliptin, Diabetic nephropathy, Kidney injury, Vascular calcification

Posted Date: February 17th, 2021

DOI: https://doi.org/10.21203/rs.3.rs-199204/v1

License: (c) (i) This work is licensed under a Creative Commons Attribution 4.0 International License.

Read Full License 


\section{Abstract}

Introduction: Dipeptidyl peptidase-4 (DPP-4) inhibitors improve glycemic control and have pleiotropic effects on kidney injury, albuminuria and vascular inflammation, especially in animal models. We plan to evaluate the effects of a potent DPP4 inhibitor (gemigliptin) on these processes in diabetic nephropathy patients.

Methods. This was a multicenter, prospective, randomized, placebo-controlled trial. A total of 201 participants were enrolled and randomly assigned to a group treated with $50 \mathrm{mg}$ gemigliptin daily with standard care of diabetes mellitus for 6 months. The changes in coronary calcium score (CAC score), cardio-ankle vascular index (CAVI), estimated glomerular filtration rate (GFR), vascular calcification and tubular renal injury markers were evaluated at baseline and 6 months.

Results. In total, 182 patients completed the study. Significant reductions in hemoglobin A1C levels were observed in both groups. The changes in CAC score, CAVI, estimated GFR and proteinuria over the 6 months of the study did not significantly differ between the groups. However, biomarkers of vascular calcification, including serum bone alkaline phosphatase, and kidney injury, including urine neutrophil gelatinase-associated lipocalin (NGAL)/Cr and urine liver fatty acid-binding protein (L-FABP)/Cr, were improved significantly in the gemigliptin treatment group compared to the control group. No serious adverse event was observed during the study.

Conclusion: Our study shows that gemigliptin significantly improves renal tubular injury biomarkers and vascular calcification in patients with diabetic nephropathy; however, gemigliptin does not affect renal function or coronary calcification compared with the control. A larger and longer follow-up will be essential to determine these beneficial effects.

\section{Clinical trials}

\section{ClinicalTrials.Gov Identifier: NCT04705506}

\section{Background}

The advantage of dipeptidyl peptidase-4 (DPP-4) inhibitors is due to their lower risk of inducing hypoglycemia in type 2 diabetic patients. ${ }^{1}$ DPP-4 inhibitor action inhibits the proteolytic enzyme DPP-4, resulting in postponement of the degradation of glucagon-like peptide I (GLP-1), thus improving glycemic control, and GLP-1 also regulates calcification of vascular smooth muscle cells through numerous pathways. ${ }^{2}$ Gemigliptin is a potent DPP-4 inhibitor that has been approved for use in patients with type 2 diabetes, and it has pleiotropic effects in addition to its glucose-lowering effect, including inhibition of lipopolysaccharide (LPS)-induced proinflammatory effects in vascular endothelial cells by attenuating NF-kappa B and c-Jun NH (2)-terminal kinase (JNK) signaling via an AMP-activated protein kinase (AMPK)-dependent mechanism. ${ }^{3} \mathrm{~A}$ recent study found that gemigliptin attenuated the calcification of the abdominal aorta as well as RUNX2 and phosphate-induced Pit-1 mRNA expression, and reactive oxygen 
species formation. Therefore, gemigliptin could benefit vascular calcification in patients with a high risk of disease, especially diabetic nephropathy.

DPP-4 inhibitors can be used safely in type 2 diabetic patients with renal impairment. Moreover, in an experimental model, DPP-4 inhibitors, especially gemigliptin, substantially decreased albuminuria and renal fibrosis in mice with unilateral ureteral obstruction ${ }^{5}$ and attenuated podocyte injury in mice with diabetic nephropathy. ${ }^{6}$ Recently, treatment with DPP-4 inhibitors attenuated kidney injury and improved acute and chronic kidney injury. ${ }^{7}$ We therefore are interested in further investigating whether gemigliptin might have a similar effect in patients with diabetic nephropathy who have a high level of vascular calcification and renal progression. We investigated the effect of gemigliptin on vascular calcification and renal injury in type 2 diabetic patients with renal involvement.

\section{Methods}

\section{Study population}

We prospectively enrolled patients from three hospitals in Bangkok, Thailand: Vajira Hospital, Police Hospital and Phramongkutklao Hospital. The study implementation and protocol were approved by the institutional review board and adhered to the Declaration of Helsinki. Informed consent was obtained from all the participants before enrollment. The inclusion criteria were type 2 diabetic patients with stage 3-4 CKD (estimated glomerular filtration rate (GFR) $15-60 \mathrm{ml} / \mathrm{min} / 1.73 \mathrm{~mL}$ ), persistent micro- or macroalbuminuria and stable glycemic control for 12 weeks. The exclusion criteria included a history of allergy to DPP4 inhibitors, concurrent infectious disease, inflammatory diseases, post kidney transplantation, and treatment with calcimimetic agents, bisphosphonates, GLP-1 receptor agonists, DPP4 inhibitors and sodium-glucose cotransporter-2 inhibitors. The criteria to withdraw were patients who had other serious side effects after inclusion, severe hypoglycemia and needed hospitalization.

\section{Study design}

This was a multicenter prospective, open-label randomized controlled trial. Patients were randomized by the study coordinator into blocks of four, the allocation was concealed, and the patients were then divided into two groups at a ratio of 1:1. A computer-generated randomization procedure in blocks of four was used. The patients were then randomized into two groups: Group 1 received $50 \mathrm{mg}$ gemigliptin per day for 6 months in addition to standard treatment, and Group 2 received standard treatment for type 2 diabetes and chronic kidney disease (CKD). Patients were scheduled for follow-up visits at months 2, 4 and 6, as shown in Figure 1. To give $80 \%$ power at $P<0.05$ to detect a difference in biomarker levels of vascular calcification after gemigliptin treatment in CKD patients, a total of 100 patients per group were required, and 182 subjects total were recruited. ${ }^{4}$

From September 2018 to October 2020, we collected baseline data from all participants for demographic characteristics, comorbid conditions and physical examination. Blood samples were collected and allowed to clot for $30 \mathrm{~min}$ at room temperature before centrifugation for 15 minutes. The serum was 
stored at $-20^{\circ} \mathrm{C}$ before assay. Urine was collected in a sterile container, centrifuged to remove particulate matter, and stored at $-20^{\circ} \mathrm{C}$ before assay. Biochemistry indexes, including complete blood count, blood urea nitrogen (BUN), creatinine, fasting plasma glucose (FPG), hemoglobin A1c (HA1C), calcium, phosphorus, albumin and 24-hour urine protein, were measured at baseline and at each study visit.

The coronary artery calcium (CAC) score; cardio-ankle vascular index (CAVI); vascular calcification biomarkers including serum osteopontin, bone alkaline phosphatase and reactive oxygen species (myeloperoxidase); and renal injury biomarkers including urine kidney injury molecule-1 (KIM-1), neutrophil gelatinase-associated lipocalin (NGAL) and liver fatty acid-binding protein (L-FABP) were determined at baseline and after 6 months. All vascular and renal injury biomarkers were tested by the quantitative sandwich enzyme immunoassay technique (R\&D Systems, USA) according to the manufacturer's instructions. Absorbance was measured at 450-570 nm using a microplate reader (SunriseTM Absorbance Reader, TECAN, Switzerland). Urine creatinine was assayed by the enzymatic method (Archetect C16000 analyzer, Tamecula, California).

\section{Coronary artery calcium scoring (CAC score)}

All patients underwent computer tomography (CT) examination using an Ingenuity CT scanner (128 slice, Philips Medical Systems, Nederland B.V.) and were acquired with axial prospective gating while breath holding. The calcium score of each lesion was calculated by using the Agatston method. ${ }^{8}$ The software package (Heart Beat Calcium Scoring, IntelliSpace Portal version 7.0; Philips Medical Systems, Nederland B.V.) automatically calculated and displayed the CT attenuation values. Agatston scores were reported for each of the 4 major coronary arteries, and the sum of these arteries was reported.

\section{Cardio-ankle vascular index (CAVI)}

We measured CAVI using a vascular screening system (Vasera VS-1500; Fukuda Denshi, Co. Ltd., Tokyo, Japan), with the patient lying supine and the head placed in the midline position. Cuffs were then applied bilaterally to the arms and ankles, and the patient was allowed to rest for $15 \mathrm{~min}$. Measurements began by obtaining the blood pressure of the right brachial artery and ankle, followed by the left brachial artery and ankle. PWV was measured by dividing the vascular length by the time taken for the pulse wave to propagate from the aortic valve to the ankle. The ankle brachial pressure index (ABI) was also calculated. CAVI was considered normal when the value was $<8$.

\section{Statistical analysis}

Continuous and categorical variables are described as the mean \pm standard deviation and numbers with parentheses. Differences between groups were compared using Student's $t$ test and the chi-square test, respectively. The number and percentage of the variables are presented by treatment group, and differences between the two treatment groups were compared using Fischer's exact test. The comparison between variables before and after treatment with gemigliptin was performed using ANOVA. Multiple linear regression analysis was performed to evaluate the effects of $\mathrm{HbA} 1 \mathrm{c}$ on renal injury markers. The 
level of significance for all statistical tests was set at 0.05. All analyses were performed using SPSS( Armonk ,NY:IBM Corp,USA) for Windows software version 22.

\section{Results}

\section{Patient characteristics}

Of the 260 patients screened for eligibility, 201 (77\%) patients were enrolled in this randomized clinical trial. Of the participants, 6 were withdrawn due to personal reasons, and 13 were excluded for other reasons. The remaining 182 participants completed the study, as shown in Figure 1.

There were 107 males and 75 females included in this study. The mean age was $62.77 \pm 9.59$ years. Sixtytwo patients $(80.52 \%)$ and 28 patients $(36.36 \%)$ had hypertension and dyslipidemia, respectively. A total of $14.29 \%(n=11)$ had a previous history of ischemic heart diseases, while 1 patient $(1.3 \%)$ had a history of peripheral arterial disease. At baseline, the 182 patients had a mean HbA1c of $8.25 \pm 1.83 \%$, a mean estimated GFR of $45.58 \pm 20.18 \mathrm{~mL} / \mathrm{min} / 1.73 \mathrm{~m}^{2}$ and a mean body mass index of $28.16 \pm 5.37 \mathrm{~kg} / \mathrm{m}^{2}$. The demographic and baseline characteristics of the groups were comparable, as shown in Table 1.

\section{Glycemic control}

Changes in the $\mathrm{HbA} 1 \mathrm{c}$ values over time are shown in Tables 2 and 3. The mean $\mathrm{HbA} 1 \mathrm{c}$ level decreased from $8.3 \pm 1.95 \%$ at baseline to $7.7 \pm 1.98 \%$ at 6 months $(P<0.001)$ in the gemigliptin group and from $8.12 \pm 1.70 \%$ at baseline to $7.98 \pm 1.88 \%$ at 6 months in the control group $(P=0.451)$ (Tables 3 and 4$)$. Significant reductions in $\mathrm{HbA} 1 \mathrm{c}$ levels were observed at month 6 in the gemigliptin group $(-0.67 \%)$ compared with the control group (-0.15\%; $\mathrm{P}=0.048)$ (Figure 2 and Table 4).

\section{Gemigliptin and vascular calcification}

The calcium content in the coronary wall as measured by CAC scores increased over time in both groups but did not significantly differ before and after the treatment period (CAC score in the gemigliptin group increased from $655.72 \pm 905.88$ to $690.93 \pm 932.89, \mathrm{P}=0.18$ and increased from $729.95 \pm 1123.98$ to $744.67 \pm 1148.90$ in the control group). The change in CAC score was also nonsignificant between the two groups (Table 4).

\section{Gemigliptin and vascular stiffness}

We measured CAVI to assess arterial stiffness. After treatment, CAVI tended to be improved in the gemigliptin group $(9.37 \pm 1.35$ at baseline vs. $9.08 \pm 1.52$ at 6 months, $P=0.08)$, and CAVI was significantly improved the control group ( $9.26 \pm 1.44$ at baseline vs. $8.69 \pm 2.06$ at 6 months, $P=0.005)$. However, the change in CAVI was not different in the two groups $(P=0.265)$ (Table 4 and Figure 3 ).

\section{Gemigliptin and markers of vascular calcification}


To examine whether gemigliptin has protective effects against vascular calcification, we examined the biochemical markers involved in vascular calcification and oxidative stress. Serum osteopontin showed no significant differences from baseline levels in both groups, and the mean changes were not significantly different between the two groups (Table 4). The major bone mineralization regulator (bone alkaline phosphatase) decreased in the gemigliptin group but increased in the control group. At 6 months, the level of bone alkaline phosphatase was significantly reduced in the gemigliptin treatment group compared to the control group $(-5.84 \pm 10.65 \mu \mathrm{g} / \mathrm{L}$ vs. $0.08 \pm 11.45 \mu \mathrm{g} / \mathrm{L}, \mathrm{P}<0.001$, respectively) (Table 4 and Figure 4). However, serum myeloperoxidase levels, which indicate oxidative stress, did not change significantly from baseline, and the mean changes did not differ between the two groups.

\section{Gemigliptin, renal fnction and proteinuria}

To verify the short-term effect of gemigliptin on estimated GFR and proteinuria, the changes in estimated GFR from a point of treatment to 6 months were compared with the standard control group. The mean changes in estimated GFR and urine protein were not significantly different from baseline in the gemigliptin and control groups.

\section{Gemigliptin and renal injury biomarkers}

We examined the effects of gemigliptin on the levels of urinary renal injury biomarkers, such as NGAL, LFABP and KIM-1. We adjusted urine biomarker concentrations by urine creatinine levels to eliminate the effects of patient hydration status. Urine NGAL tended to decrease but did not reach statistical significance in the gemigliptin group ( $387.9 \pm 1094.02 \mathrm{ng} / \mathrm{mg}$ creatinine at baseline vs. $316.37 \pm 679.96$ $\mathrm{ng} / \mathrm{mg}$ creatinine at the end of the study, $\mathrm{P}=0.412$ ) (Table 2), but urine NGAL significantly increased in the control group $(333.67 \pm 627.23 \mathrm{ng} / \mathrm{mg}$ creatinine at baseline vs. $590.95 \pm 1252.05 \mathrm{ng} / \mathrm{mg}$ creatinine at the end of the study, $\mathrm{P}=0.024$ ) (Table 3 ). The change in urine NGAL between the two groups was significantly different $(-71.53 \pm 837.30 \mathrm{ng} / \mathrm{mg}$ creatinine in the gemigliptin group vs $257.28 \pm 1,047.29$ $\mathrm{ng} / \mathrm{mg}$ creatinine in the control group, $\mathrm{P}=0.020$ ) (Table 4 and Figure 5).

Urine LFABP decreased significantly in the gemigliptin group $(91.32 \pm 146.92 \mu \mathrm{g} / \mathrm{mg}$ creatinine at baseline vs. $37.16 \pm 68.47 \mu \mathrm{g} / \mathrm{mg}$ creatinine at the end of study, $P<0.001)$, while urine LFABP was not significantly changed $(48.86 \pm 67.14 \mu \mathrm{g} / \mathrm{mg}$ creatinine at baseline vs. $55.47 \pm 86.31 \mu \mathrm{g} / \mathrm{mg}$ creatinine at the end of study, $\mathrm{P}=0.488$ ). The degree of change in the control group was significantly different from that in the gemigliptin group $(-54.17 \pm 141.64 \mu \mathrm{g} / \mathrm{mg}$ creatinine in the gemigliptin group vs $6.6 \pm 88.94$ $\mu \mathrm{g} / \mathrm{mg}$ creatinine in the control group, $\mathrm{P}=0.001$ ) (Table 4 and Figure 6 ). The effect of HbA1c on lowering renal biomarkers was not significant according to multiple linear regression analysis (Supplemental file).

Urine KIM-1 decreased in both groups, but the change in urine KIM-1 did not differ between the groups $(-0.28 \pm 1.15 \mathrm{ng} / \mathrm{mg}$ creatinine in the gemigliptin group vs $-0.50 \pm 0.90 \mathrm{ng} / \mathrm{mg}$ creatinine in the control group, $\mathrm{P}=0.156$ ).(Figure 7)

The comparison of significant parameters are shown in supplemental file 


\section{Adverse events}

Adverse events due to gemigliptin was rare. The most commonly reported adverse events from previous data were hypoglycemia, upper respiratory tract infection, urinary tract infection, nasopharyngitis, headache, diarrhea, arthralgia, hypertension, and cough. ${ }^{9}$ However, we found only one case that reported palpitation after gemigliptin administration. Finally, the patient asked for withdrawal from the trial.

\section{Discussion}

Many investigators have studied the pleiotropic properties of DPP-4 inhibitors to highlight their potential benefits in various diseases. At present, there are scarce data evaluating the effects of DPP-4 inhibitors on vascular calcification in vivo. We chose gemigliptin due to its unique characteristics, including its action as a highly competitive and selective DPP-4 inhibitor. Then, we evaluated the effect of gemigliptin on vascular calcification by using CAC scores and CAVI. After a follow-up period of 6 months, the CAC score and CAVI were not different between the two groups. This may be because the period of observation was too short. However, the markers of vascular calcification and bone alkaline phosphatase (BALP) decreased significantly in the gemigliptin group compared with the control group. BALP is a sensitive and specific marker for osteoblast activity and bone formation. Alkaline phosphatase stimulates mineralization mainly through the modulation of the balance between inorganic phosphate and inorganic pyrophosphate and has a role in cardiovascular remodeling. Yan et al. ${ }^{10}$ showed that BALP was an independent risk factor for abdominal aortic calcification and suggested a strong relationship between BALP and vascular calcification in a dialysis population. Shantouf et al. ${ }^{11}$ reported a significant association between serum alkaline phosphatase and coronary artery calcification in maintenance hemodialysis. Taken together, the decrease in BALP and tendency to increase osteopontin levels by gemigliptin in our study might suggest the potential role of DPP4 inhibitors in protecting against vascular calcification in the long term.

The role of DPP-4 inhibitors in renal disease is not fully understood. DPP-4 is highly expressed in proximal renal tubular cells and has proteolytic activity by involving the extracellular catabolism of proteins in the kidney, such as proline-containing peptide. DPP-4 inhibition is likely to alter the degradation/regulation of peptides in the lumen and thus influence tubular structure and function in diabetes. ${ }^{12}$ In addition to the glucose-lowering effects of DPP-4 inhibitors and the tissue-protective effects of DPP-4 inhibition have been demonstrated in particular ischemia-reperfusion injury, diabetic kidney disease and CKD. Kim et al. ${ }^{13}$ reported that gemigliptin treatment led to a reduction in apoptosis, inflammation, and oxidative stress in a murine model of adriamycin-induced nephropathy. Choi et al. ${ }^{14}$ showed that gemigliptin attenuated cisplatin-induced renal dysfunction in mice. The mechanisms were possibly due to inhibition of the apoptotic death of renal tubular cells and inflammatory responses. Of interest, we studied biomarkers of early kidney injury, such as KIM-1, NGAL and LFABP, which are not only more sensitive than serum creatinine for the identification of acute kidney injury but can also indicate specific damage to the proximal tubule. ${ }^{15}$ Urine NGAL and urine LFABP were significantly decreased after 
gemigliptin treatment. To our knowledge, this is the first study to show that DPP4 inhibitors alleviate kidney injury through the use of new biomarkers. Urine NGAL has been shown to be increased in many pathologic conditions, including diabetic kidney disease. ${ }^{16}$ The level of urinary NGAL appears to increase beginning in the early phase of diabetic nephropathy, and the level is independently associated with albuminuria. ${ }^{16,17}$ The novel finding of this study is that urine NGAL was significantly decreased after gemigliptin treatment, which may indicate that gemigliptin helps ameliorate tubulointerstitial damage. Indeed, the evolution of the estimated GFR was not significantly changed. A short follow-up time may influence the therapeutic response. NGAL is considered a biomarker to monitor disease progression.

L-FABP regulates fatty acid uptake and intracellular transport, and the excretion rate of this protein is associated with tubulointerstitial structural damage. ${ }^{18}$ Therefore, L-FABP can be used to identify patients with a high susceptibility to renal stress. Increased urine concentrations of L-FABP have been observed in diabetic nephropathy. ${ }^{19}$ Indeed, urine L-FABP was elevated at the very early stage of diabetic nephropathy, even before any clinical signs of glomerular damage were detectable, and tubular damage, albuminuria and end-stage renal disease were independently predicted. ${ }^{20}$ In this study, urine L-FABP markedly decreased after gemigliptin treatment. These results suggest that gemigliptin could reduce kidney injury and thus reduce the rate of renal disease progression. Long-term prospective follow-up may demonstrate whether eGFR is affected.

KIM-1 is a transmembrane protein that is markedly upregulated at the renal proximal tubule after injury. ${ }^{21}$ Urinary levels of KIM-1 were significantly elevated in diabetic patients, indicating the existence of diabetic tubular damage at the early stage of diabetic nephropathy. ${ }^{22}$ The expression of KIM-1 is mainly upregulated in proximal tubule cells both in rodents and in humans. ${ }^{23}$ We found that urine KIM-1 was decreased significantly in both groups. The difference in urine KIM-1 was similar in both groups. However, KIM-1 may not be as sensitive of a marker as urine LFABP and NGAL to detect the favorable effects of gemigliptin. The mechanism by which gemigliptin significantly reduces renal injury may be related to the antifibrotic, antiapoptotic and anti-inflammatory action of DPP4 inhibitors independent of their antiproteinuric and glucose-lowering effects. The markers that we chose to study are sensitive and established indicators for subtle insults to the kidney before measurable functional decline. These urinary markers were elevated before microalbuminuria was observed; thus, their values might be altered due to the changes in estimated GFR.

There were some limitations to our study. First, patients were followed for a relatively short duration (6 months). The antiatherosclerotic effects of gemigliptin, such as the effects on the CAC score and CAVI, could not be clearly demonstrated. The exposure to the study drug may not have been long enough to reverse the effects of years of pro-atherosclerotic processes in patients with a median duration of diabetes mellitus of more than 10 years; therefore, the study does not exclude the possibility of either benefit or risk with a longer duration of gemigliptin therapy. A longer study period needs to be continued. Second, it is not surprising that DPP-4 inhibitors significantly lower glucose levels. However, we used multiple logistic regression analysis to adjust for this factor. 
In conclusion, our study demonstrated that gemigliptin improved glycemic control, vascular calcification markers and kidney injury biomarkers. Urinary excretion of these markers is an early, sensitive and specific marker for diabetic nephropathy that helps denote the beneficial effect of gemigliptin. In addition to the rare side effects of this class of DPP-4 inhibitors, their pleiotropic actions help retard tubular injury and diabetic nephropathy progression, which is of great clinical relevance. Additional large and long-term studies are needed to confirm their clinical benefit and utility.

\section{Abbreviations}

DN: Diabetic nephropathy

eGFR: Estimated glomerular filtration rate

KIM-1: Kidney injury molecule-1

L-FABP: Liver-type fatty acid binding protein

NGAL: Neutrophil gelatinase associated lipocalin

UACR: Urinary albumin-creatinine ratio

AAC : Abdominal aortic calcification

\section{Declarations}

The study and protocol were approved by the Navamindradhiraj institutional review board and adhered to the Declaration of Helsinki. Informed consent was obtained from all the participants before enrollment. The authors approved for the publication in this journal.

\section{Competeing interestes}

The authors declare no competing interests.

\section{Author's contribution}

TT Design the protocol,collect data,wrote paper,conceptualize

BS set up database,analyzed data,wrote paper

SS collect data

TC collect data

AS Interpretation of CAVI data 
TS Interpretation of CAVI data

All authors read and approved the final manuscript.

\section{Acknowledgements}

The authors gratefully acknowledge the assistance of Lt. Rattanawan Dispan for the laboratory experiments and Ms. Worachanee Imjaijit for statistical analysis.

\section{Funding information}

This trial was funded by the Navamindradhiraj University research fund, and the LG Life Science Company provided the medicine (gemigliptin) for the whole study.

\section{Role of the funding source}

The LG life Science has no role in study design, the collection ,analysis and interpretation of data.in the writing of the report together with the publication

\section{Data availability}

The datasets generated and analysed during the current study are available in the figshare repository [https://figshare.com/s/059283ae1fd803a85939]

\section{References}

1. Panchapakesan U, Pollock C. The role of dipeptidyl peptidase - 4 inhibitors in diabetic kidney disease. Front Immunol. 2015;6:443.

2. Hwang HJ, Chung HS, Jung TW, et al. The dipeptidyl peptidase-IV inhibitor inhibits the expression of vascular adhesion molecules and inflammatory cytokines in HUVECs via Akt- and AMPK-dependent mechanisms. Mol Cell Endocrinol. 2015;405:25-34.

3. Gutch M, Joshi A, Kumar S, et al. Gemigliptin: Newer promising gliptin for type 2 diabetes mellitus. Indian J Endocrinol Metab. 2017;21:898-902.

4. Choi SY, Ryu HM, Oh EJ, et al. Dipeptidyl peptidase-4 inhibitor gemigliptin protects against vascular calcification in an experimental chronic kidney disease and vascular smooth muscle cells. PLoS One. 2017;12:e0180393.

5. Min HS, Kim JE, Lee MH, et al. Dipeptidyl peptidase IV inhibitor protects against renal interstitial fibrosis in a mouse model of ureteral obstruction. Lab Invest. 2014;94:598-607.

6. Jung E, Kim J, Ho Kim S, et al. Gemigliptin improves renal function and attenuates podocyte injury in mice with diabetic nephropathy. Eur J Pharmacol. 2015;761:116-124.

7. Joo KW, Kim S, Ahn SY, et al. Dipeptidyl peptidase IV inhibitor attenuates kidney injury in rat remnant kidney. BMC Nephrol. 2013;14:98. 
8. Agatston AS, Janowitz WR, Hildner FJ, et al. Quantification of coronary artery calcium using ultrafast computed tomography. J Am Coll Cardiol. 1990;15:827-832.

9. Kim SH, Yoo JH, Lee WJ, et al. Gemigliptin: an update of its clinical use in the management of type 2 diabetes mellitus. Diabetes Metab J. 2016;40:339-353.

10. Yan J, Li L, Zhang M, et al. Circulating bone-specific alkaline phosphatase and abdominal aortic calcification in maintenance hemodialysis patients. Biomark Med. 2018;12:1231-1239.

11. Shantouf R, Kovesdy CP, Kim Y, et al. Association of serum alkaline phosphatase with coronary artery calcification in maintenance hemodialysis patients. Clin J Am Soc Nephrol. 2009;4:1106-1114.

12. Tiruppathi $C$, Miyamoto $Y$, Ganapathy $V$, et al. Hydrolysis and transport of proline-containing peptides in renal brush-border membrane vesicles from dipeptidyl peptidase IV-positive and dipeptidyl peptidase IV-negative rat strains. J Biol Chem. 1990;265:1476-1483.

13. Kim DR, Lee SY, Kim JS, et al. Ameliorating effect of gemigliptin on renal injury in murine adriamycininduced nephropathy. Biomed Res Int. 2017;2017:7275109.

14. Choi SH, Leem J, Lee IK. Protective effects of gemigliptin, a dipeptidyl peptidase-4 inhibitor, against cisplatin-induced nephrotoxicity in mice. Mediators Inflamm. 2017;2017:4139439.

15. Satirapoj B. Tubulointerstitial biomarkers for diabetic nephropathy. J Diabetes Res. 2018;2018:2852398.

16. Wu J, Shao X, Lu K, et al. Urinary RBP and NGAL levels are associated with nephropathy in patients with type 2 diabetes. Cell Physiol Biochem. 2017;42:594-602.

17. Wu C, Wang Q, Lv C, et al. The changes of serum sKlotho and NGAL levels and their correlation in type 2 diabetes mellitus patients with different stages of urinary albumin. Diabetes Res Clin Pract. 2014;106:343-350.

18. Kamijo A, Sugaya $T$, Hikawa A, et al. Urinary excretion of fatty acid-binding protein reflects stress overload on the proximal tubules. Am J Pathol. 2004;165:1243-1255.

19. Suzuki K, Babazono T, Murata $\mathrm{H}$, et al. Clinical significance of urinary liver-type fatty acid-binding protein in patients with diabetic nephropathy. Diabetes Care. 2005;28:2038-2039.

20. Nielsen SE, Sugaya T, Hovind P, et al. Urinary liver-type fatty acid-binding protein predicts progression to nephropathy in type 1 diabetic patients. Diabetes Care. 2010;33:1320-1324.

21. Han WK, Bailly V, Abichandani R, et al. Kidney Injury Molecule-1 (KIM-1): a novel biomarker for human renal proximal tubule injury. Kidney Int. 2002;62:237-244.

22. Satirapoj B, Pooluea P, Nata N, et al. Urinary biomarkers of tubular injury to predict renal progression and end stage renal disease in type 2 diabetes mellitus with advanced nephropathy: a prospective cohort study. J Diabetes Complications. 2019;33:675-681.

23. Ichimura T, Bonventre JV, Bailly V, et al. Kidney injury molecule-1 (KIM-1), a putative epithelial cell adhesion molecule containing a novel immunoglobulin domain, is up-regulated in renal cells after injury. J Biol Chem. 1998;273:4135-4142. 


\section{Tables}

Table 1 Baseline characteristics of the two groups

\begin{tabular}{|llll|}
\hline & $\begin{array}{l}\text { Gemigliptin } \\
(\mathrm{N}=94)\end{array}$ & $\begin{array}{l}\text { Control } \\
(\mathrm{N}=88)\end{array}$ & P-value \\
\hline Male (\%) & $55(58.51 \%)$ & $52(59.09 \%)$ & 0.937 \\
\hline Age (years) & $62.9 \pm 8.7$ & $62.7 \pm 10.5$ & 0.881 \\
\hline Duration of diabetes (years) & $13(1-20)$ & $11(1-40)$ & 0.493 \\
\hline Hypoglycemic agents (\%) & & & \\
\hline Metformin (\%) & 56.04 & 54.02 & 0.786 \\
\hline Sulfonylurea (\%) & 68.13 & 56.82 & 0.118 \\
\hline Glitazone (\%) & 41.76 & 36.78 & 0.497 \\
\hline SGLT2 inhibitors (\%) & 0 & 0 & 0 \\
\hline Insulin (\%) & 7.69 & 10.34 & 0.536 \\
\hline Body weight (kg) & $75.1 \pm 16.5$ & $72.7 \pm 14.1$ & 0.296 \\
\hline Body mass index (kg/m $\left.{ }^{2}\right)$ & $28.5 \pm 5.9$ & $27.8 \pm 4.7$ & 0.361 \\
\hline Hypertension (\%) & $78(83.3 \%)$ & $67(77.1 \%)$ & 0.495 \\
\hline Dyslipidemia (\%) & $33(35.7 \%)$ & $32(37.1 \%)$ & 0.897 \\
\hline Peripheral vascular disease (\%) & 0 & $2(2.9 \%)$ & 0.455 \\
\hline Ischemic heart disease (\%) & $6(7.1 \%)$ & $20(22.9 \%)$ & 0.500 \\
\hline
\end{tabular}

Data are presented as the mean \pm SD or percentage.

Table 2 Changes in clinical characteristics and laboratory indexes in the gemigliptin group from baseline to month 6 


\begin{tabular}{|c|c|c|c|c|}
\hline & Baseline & Month 6 & $\begin{array}{l}\text { Mean } \\
\text { difference }\end{array}$ & $\begin{array}{l}\text { P- } \\
\text { value }\end{array}$ \\
\hline Body weight (kg) & $75.16 \pm 16.6$ & $75.37 \pm 16.71$ & $0.22 \pm 4.20$ & 0.622 \\
\hline Body mass index $\left(\mathrm{kg} / \mathrm{m}^{2}\right)$ & $28.52 \pm 6.01$ & $28.59 \pm 5.94$ & $0.07 \pm 1.57$ & 0.673 \\
\hline Mean arterial blood pressure & $112.08 \pm 14.68$ & $108.76 \pm 16.59$ & $-3.31 \pm 18.02$ & 0.080 \\
\hline Hemoglobin (g/dL) & $12.44 \pm 1.80$ & $12.17 \pm 1.75$ & $-0.26 \pm 0.99$ & 0.018 \\
\hline $\begin{array}{l}\text { Estimated GFR }(\mathrm{mL} / \mathrm{min} / 1.73 \\
\left.\mathrm{m}^{2}\right)\end{array}$ & $46.83 \pm 19.14$ & $45.8 \pm 21.27$ & $-1.03 \pm 10.00$ & 0.340 \\
\hline $\mathrm{BUN}(\mathrm{mg} / \mathrm{dL})$ & $26.32 \pm 10.18$ & $27.58 \pm 12.99$ & $1.27 \pm 8.75$ & 0.189 \\
\hline Serum creatinine (mg/dL) & $1.62 \pm 0.59$ & $1.70 \pm 0.70$ & $0.09 \pm 0.33$ & 0.018 \\
\hline Fasting plasma glucose (mg/dL) & $185.36 \pm 56.91$ & $152.02 \pm 60.14$ & $-33.34 \pm 59.40$ & $<0.001$ \\
\hline Hemoglobin A1C (\%) & $8.37 \pm 1.95$ & $7.7 \pm 1.98$ & $-0.67 \pm 1.59$ & $<0.001$ \\
\hline Serum potassium (mEq/L) & $4.49 \pm 0.50$ & $4.40 \pm 0.48$ & $-0.08 \pm 0.49$ & 0.141 \\
\hline Serum magnesium (mg/dL) & $1.92 \pm 0.30$ & $2.14 \pm 1.26$ & $0.22 \pm 1.19$ & 0.128 \\
\hline Serum calcium (mg/dL) & $9.4 \pm 0.52$ & $9.14 \pm 0.95$ & $-0.26 \pm 1.03$ & 0.037 \\
\hline Serum phosphate $(\mathrm{mg} / \mathrm{dL})$ & $3.88 \pm 0.70$ & $3.75 \pm 0.76$ & $-0.14 \pm 0.69$ & 0.105 \\
\hline Serum albumin (g/dL) & $3.84 \pm 0.52$ & $3.87 \pm 0.48$ & $0.04 \pm 0.35$ & 0.379 \\
\hline Urine protein, 24 hour (g/day) & $1.77 \pm 3.15$ & $1.76 \pm 3.39$ & $-0.01 \pm 2.96$ & 0.983 \\
\hline Coronary artery calcium score & $\begin{array}{l}655.72 \pm \\
905.88\end{array}$ & $690.93 \pm 932.89$ & $35.21 \pm 252.88$ & 0.180 \\
\hline CAVI & $9.37 \pm 1.35$ & $9.08 \pm 1.52$ & $-0.28 \pm 1.58$ & 0.085 \\
\hline Serum myeloperoxidase $(\mu \mathrm{g} / \mathrm{L})$ & $\begin{array}{l}641.58 \pm \\
232.89\end{array}$ & $620.76 \pm 244.38$ & $\begin{array}{l}-20.82 \pm \\
298.75\end{array}$ & 0.501 \\
\hline Serum osteopontin (ng/mL) & $\begin{array}{l}2438.58 \pm \\
1439.96\end{array}$ & $\begin{array}{l}2749.25 \pm \\
1506.93\end{array}$ & $\begin{array}{l}310.67 \pm \\
2222.67\end{array}$ & 0.179 \\
\hline $\begin{array}{l}\text { Serum bone alkaline phosphate } \\
(\mu \mathrm{g} / \mathrm{L})\end{array}$ & $18.15 \pm 11.62$ & $12.30 \pm 6.81$ & $-5.85 \pm 10.65$ & $<0.001$ \\
\hline Urine NGAL (ng/mg creatinine) & $\begin{array}{l}387.9 \pm \\
1094.02\end{array}$ & $316.37 \pm 679.96$ & $\begin{array}{l}-71.53 \pm \\
837.30\end{array}$ & 0.412 \\
\hline Urine Kim (ng/mg creatinine) & $0.90 \pm 0.97$ & $0.63 \pm 0.82$ & $-0.28 \pm 1.15$ & 0.023 \\
\hline Urine LFABP ( $\mu \mathrm{g} / \mathrm{mg}$ creatinine) & $91.32 \pm 146.92$ & $37.16 \pm 68.47$ & $\begin{array}{l}-54.17 \pm \\
141.64\end{array}$ & $<0.001$ \\
\hline
\end{tabular}


Data are presented as the mean \pm SD.

CAVI, cardio-ankle vascular index; LFABP, liver acid-binding protein; NGAL, neutrophil gelatinaseassociated lipocalin; Kim-1, kidney injury molecule-1.

Table 3 Changes in clinical characteristics and laboratory indexes in the control group from baseline to month 6 


\begin{tabular}{|c|c|c|c|c|}
\hline & Baseline & Month 6 & $\begin{array}{l}\text { Mean } \\
\text { difference }\end{array}$ & $\begin{array}{l}\text { P- } \\
\text { value }\end{array}$ \\
\hline Body weight (kg) & $72.74 \pm 14.24$ & $72.51 \pm 15.20$ & $-0.23 \pm 4.70$ & 0.654 \\
\hline Body mass index $\left(\mathrm{kg} / \mathrm{m}^{2}\right)$ & $27.72 \pm 4.61$ & $27.63 \pm 4.92$ & $-0.09 \pm 1.75$ & 0.619 \\
\hline Mean arterial blood pressure & $115.98 \pm 17.41$ & $116.32 \pm 19.12$ & $0.34 \pm 17.33$ & 0.856 \\
\hline Hemoglobin (g/dL) & $12.35 \pm 1.94$ & $12.1 \pm 1.98$ & $-0.25 \pm 0.88$ & 0.012 \\
\hline $\begin{array}{l}\text { Estimated GFR }(\mathrm{mL} / \mathrm{min} / 1.73 \\
\left.\mathrm{m}^{2}\right)\end{array}$ & $44.27 \pm 21.25$ & $42.36 \pm 22.36$ & $-1.90 \pm 10.91$ & 0.116 \\
\hline $\mathrm{BUN}(\mathrm{mg} / \mathrm{dL})$ & $27.48 \pm 13.33$ & $30.28 \pm 15.44$ & $2.79 \pm 8.30$ & 0.004 \\
\hline Serum creatinine (mg/dL) & $1.75 \pm 0.69$ & $1.88 \pm 0.95$ & $0.14 \pm 0.42$ & 0.004 \\
\hline Fasting plasma glucose (mg/dL) & $167.19 \pm 51.07$ & $154.52 \pm 58.65$ & $-12.67 \pm 62.46$ & 0.065 \\
\hline Hemoglobin A1C (\%) & $8.12 \pm 1.70$ & $7.98 \pm 1.88$ & $-0.15 \pm 1.70$ & 0.451 \\
\hline Serum potassium (mEq/L) & $4.59 \pm 0.75$ & $4.47 \pm 0.50$ & $-0.12 \pm 0.77$ & 0.183 \\
\hline Serum magnesium (mg/dL) & $1.98 \pm 0.27$ & $1.92 \pm 0.24$ & $-0.05 \pm 0.26$ & 0.128 \\
\hline Serum calcium (mg/dL) & $9.27 \pm 0.51$ & $9.25 \pm 0.46$ & $-0.02 \pm 0.52$ & 0.729 \\
\hline Serum phosphate $(\mathrm{mg} / \mathrm{dL})$ & $4.05 \pm 0.85$ & $3.95 \pm 0.86$ & $-0.10 \pm 0.88$ & 0.316 \\
\hline Serum albumin (g/dL) & $3.78 \pm 0.46$ & $3.74 \pm 0.50$ & $-0.04 \pm 0.28$ & 0.239 \\
\hline Urine protein, 24 hour (g/day) & $0.89 \pm 1.60$ & $1.39 \pm 2.62$ & $0.49 \pm 2.22$ & 0.060 \\
\hline Coronary artery calcium score & $\begin{array}{l}729.95 \pm \\
1123.98\end{array}$ & $\begin{array}{l}744.67 \pm \\
1148.90\end{array}$ & $14.73 \pm 161.66$ & 0.395 \\
\hline CAVI & $9.26 \pm 1.44$ & $8.69 \pm 2.06$ & $-0.57 \pm 1.81$ & 0.005 \\
\hline Serum myeloperoxidase $(\mu \mathrm{g} / \mathrm{L})$ & $\begin{array}{l}653.06 \pm \\
208.78\end{array}$ & $655.67 \pm 232.65$ & $2.61 \pm 295.2$ & 0.934 \\
\hline Serum osteopontin (ng/mL) & $\begin{array}{l}2442.31 \pm \\
1368.92\end{array}$ & $\begin{array}{l}2668.69 \pm \\
1656.47\end{array}$ & $\begin{array}{l}226.39 \pm \\
2057.35\end{array}$ & 0.305 \\
\hline $\begin{array}{l}\text { Serum bone alkaline phosphate } \\
(\mu \mathrm{g} / \mathrm{L})\end{array}$ & $15.59 \pm 11.06$ & $15.68 \pm 8.24$ & $0.08 \pm 11.45$ & 0.947 \\
\hline Urine NGAL (ng/mg creatinine) & $\begin{array}{l}333.67 \pm \\
627.23\end{array}$ & $\begin{array}{l}590.95 \pm \\
1252.05\end{array}$ & $\begin{array}{l}257.28 \pm \\
1047.29\end{array}$ & 0.024 \\
\hline Urine Kim (ng/mg creatinine) & $1.11 \pm 1.07$ & $0.61 \pm 0.74$ & $-0.50 \pm 0.90$ & $<0.001$ \\
\hline Urine LFABP ( $\mu \mathrm{g} / \mathrm{mg}$ creatinine) & $48.86 \pm 67.14$ & $55.47 \pm 86.31$ & $6.6 \pm 88.94$ & 0.488 \\
\hline
\end{tabular}

Data are presented as the mean \pm SD . 
CAVI, cardio-ankle vascular index; LFABP, liver acid-binding protein; NGAL, neutrophil gelatinaseassociated lipocalin; Kim-1, kidney injury molecule-1.

Table 4 Comparison of mean changes in various parameters from baseline to month 6 between the two groups 


\begin{tabular}{|c|c|c|c|c|}
\hline Mean change & $\begin{array}{l}\text { Gemigliptin } \\
(\mathrm{N}=94)\end{array}$ & $\begin{array}{l}\text { Control } \\
(\mathrm{N}=88)\end{array}$ & $95 \% \mathrm{Cl}$ & $\begin{array}{l}\mathrm{P}- \\
\text { value }\end{array}$ \\
\hline Body weight (kg) & $0.22 \pm 4.20$ & $-0.23 \pm 4.70$ & $-0.87,1.75$ & 0.506 \\
\hline Body mass index $\left(\mathrm{kg} / \mathrm{m}^{2}\right)$ & $0.07 \pm 1.57$ & $-0.09 \pm 1.75$ & $-0.33,0.65$ & 0.512 \\
\hline Mean arterial blood pressure & $-3.31 \pm 18.02$ & $0.34 \pm 17.33$ & $-8.89,1.59$ & 0.171 \\
\hline Hemoglobin (g/dL) & $-0.26 \pm 0.99$ & $-0.25 \pm 0.88$ & $-0.30,0.28$ & 0.952 \\
\hline Estimated GFR $\left(\mathrm{mL} / \mathrm{min} / 1.73 \mathrm{~m}^{2}\right)$ & $-1.03 \pm 10.00$ & $-1.90 \pm 10.91$ & $-2.29,4.04$ & 0.587 \\
\hline BUN (mg/dL) & $1.27 \pm 8.75$ & $2.79 \pm 8.30$ & $-4.16,1.10$ & 0.254 \\
\hline Serum creatinine (mg/dL) & $0.09 \pm 0.33$ & $0.14 \pm 0.42$ & $-0.16,0.06$ & 0.389 \\
\hline Fasting plasma glucose (mg/dL) & $-33.34 \pm 59.40$ & $-12.67 \pm 62.46$ & $-38.96,-2.38$ & 0.027 \\
\hline Hemoglobin A1C (\%) & $-0.67 \pm 1.59$ & $-0.15 \pm 1.70$ & $-1.05,-0.01$ & 0.048 \\
\hline Serum potassium (mEq/L) & $-0.08 \pm 0.49$ & $0.44 \pm 4.02$ & $-0.96,1.44$ & 0.734 \\
\hline Serum magnesium (mg/dL) & $0.22 \pm 1.19$ & $-0.05 \pm 0.26$ & $-0.01,0.56$ & 0.057 \\
\hline Serum calcium (mg/dL) & $-0.26 \pm 1.03$ & $-0.02 \pm 0.52$ & $-0.50,0.02$ & 0.073 \\
\hline Serum phosphate (mg/dL) & $-0.14 \pm 0.69$ & $-0.10 \pm 0.88$ & $-0.29,0.23$ & 0.800 \\
\hline Serum albumin (g/dL) & $0.04 \pm 0.35$ & $-0.04 \pm 0.28$ & $-0.03,0.18$ & 0.152 \\
\hline Urine protein, 24 hour (g/day) & $-0.01 \pm 2.96$ & $0.49 \pm 2.22$ & $-1.35,0.35$ & 0.246 \\
\hline Coronary artery calcium score & $35.21 \pm 252.88$ & $14.73 \pm 161.66$ & $-42.07,83.03$ & 0.519 \\
\hline CAVI & $-0.28 \pm 1.58$ & $-0.57 \pm 1.81$ & $-0.22,0.79$ & 0.265 \\
\hline Serum myeloperoxidase $(\mu \mathrm{g} / \mathrm{L})$ & $-20.82 \pm 298.75$ & $\begin{array}{l}226.39 \pm \\
2057.35\end{array}$ & $\begin{array}{l}-543.35 \\
711.91\end{array}$ & 0.596 \\
\hline Serum osteopontin (ng/mL) & $\begin{array}{l}310.67 \pm \\
2,222.67\end{array}$ & $\begin{array}{l}226.39 \pm \\
2057.35\end{array}$ & $\begin{array}{l}-543.35 \\
711.91\end{array}$ & 0.791 \\
\hline $\begin{array}{l}\text { Serum bone alkaline phosphate } \\
(\mu \mathrm{g} / \mathrm{L})\end{array}$ & $-5.84 \pm 10.65$ & $0.08 \pm 11.45$ & $-9.16,-2.69$ & $<0.001$ \\
\hline Urine NGAL (ng/mg creatinine) & $-71.53 \pm 837.30$ & $\begin{array}{l}257.28 \pm \\
1,047.29\end{array}$ & $\begin{array}{l}-606.19 \\
-51.43\end{array}$ & 0.020 \\
\hline Urine Kim (ng/mg creatinine) & $-0.28 \pm 1.15$ & $-0.50 \pm 0.90$ & $-0.08,0.52$ & 0.156 \\
\hline Urine LFABP ( $\mu \mathrm{g} / \mathrm{mg}$ creatinine) & $-54.17 \pm 141.64$ & $6.6 \pm 88.94$ & $-95.30,-26.24$ & 0.001 \\
\hline
\end{tabular}

Data are presented as the mean \pm SD and $95 \% \mathrm{Cl}$. 
CAVI, cardio-ankle vascular index; LFABP, liver acid-binding protein; NGAL, neutrophil gelatinaseassociated lipocalin; Kim-1, kidney injury molecule-1.

\section{Figures}

\section{Patients with Type 2 Diabetes Mellitus}

$$
\mathrm{n}=340
$$
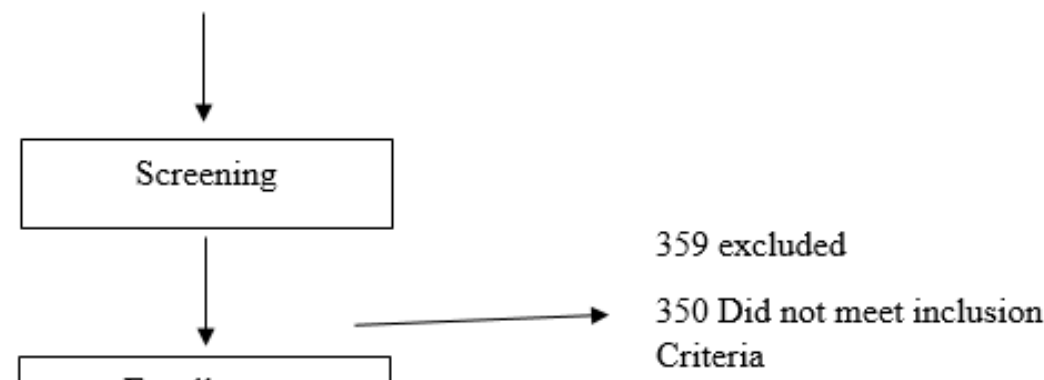

Enrollment 9 Refused To participate

\section{Randomized}

Biochemical examination

CAC Scan

Gemigliptin

$\mathrm{N}=94$ Completed Study

3 withdraw

1 death

1 Change hospital

1 intolerance to medication

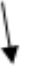

Follow up every 2 months

for 6 months
Control

$\mathrm{N}=88$ Completed Study

2 withdraw

3 death

7 loss Follow up

1 Transfer to Hemodialysis

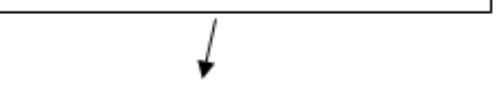

Follow up every 2 months

for 6 months

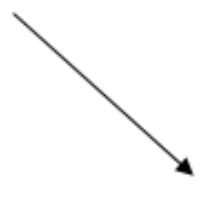

Laboratory

examination

CAC , CAVI

\section{Figure 1}


Enrollment, Follow up and Vital Status
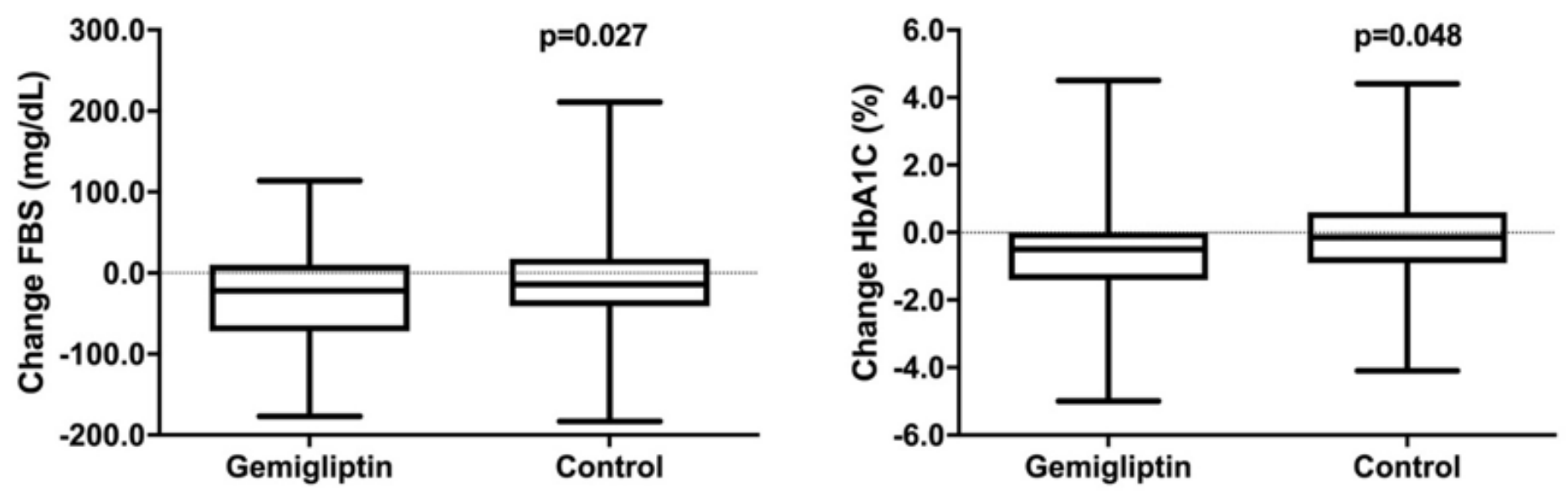

Figure 2

Shown are Box Plot indicated changes in fasting blood sugar (Panel A) and glycated hemoglobin (Panel B) during the trial period.Data was set on the basis of scheduled visits.

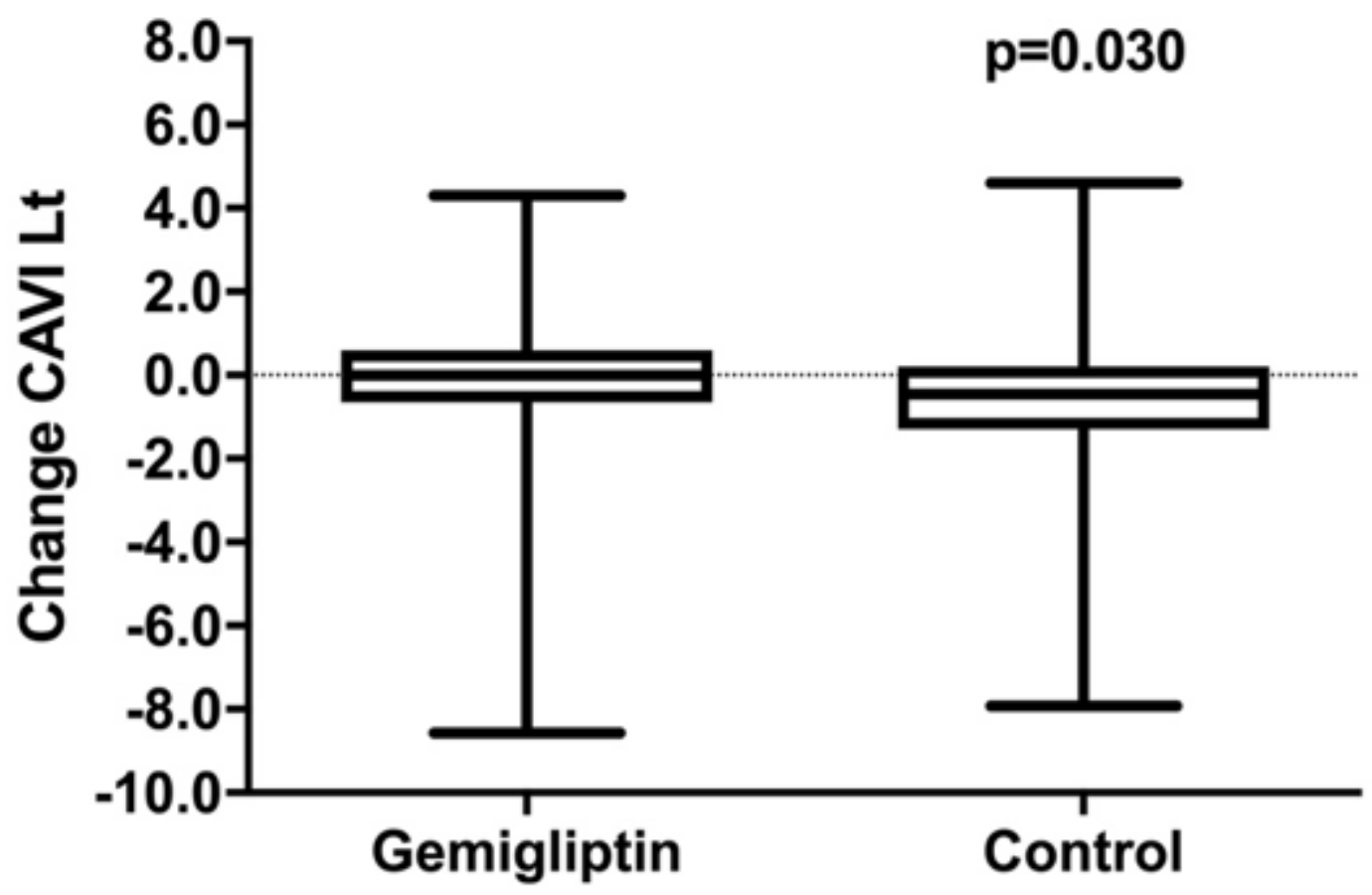

Figure 3

Changes in CAVI I both groups at baseline and at the end of study 


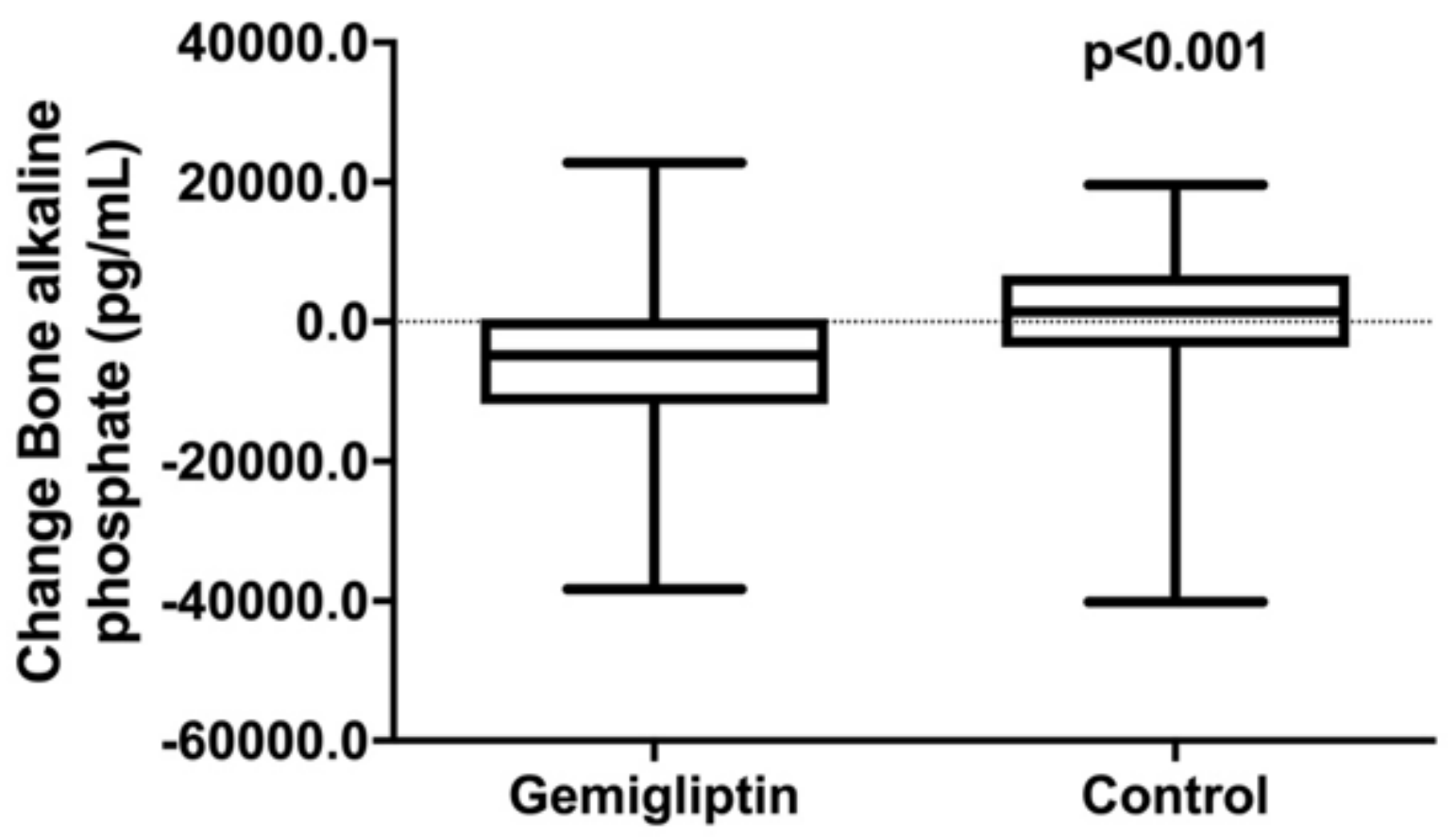

Figure 4

Changes in Bone alkaline phosphatase in both groups at baseline and at the end of study
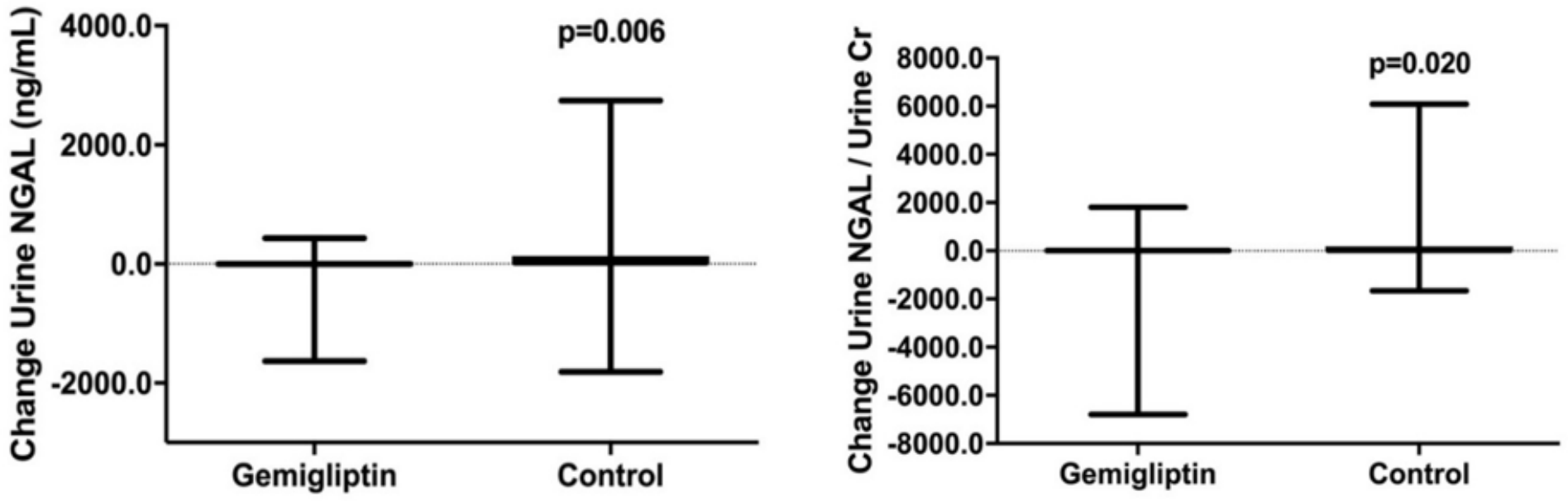

Figure 5

Comparisons between urine NGAL and urine NGAL factor for urine creatinine in both groups at baseline and at the end of study 

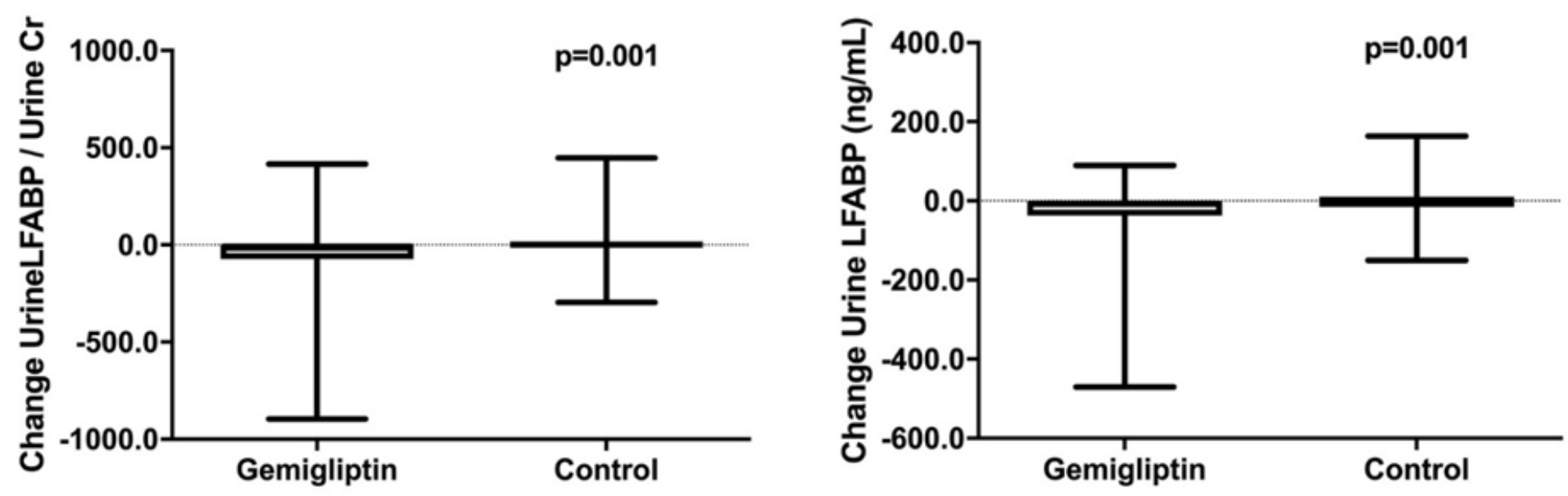

Figure 6

Comparisons between urine LFABP and urine LFABP factor for urine creatinine in both groups at baseline and at the end of study
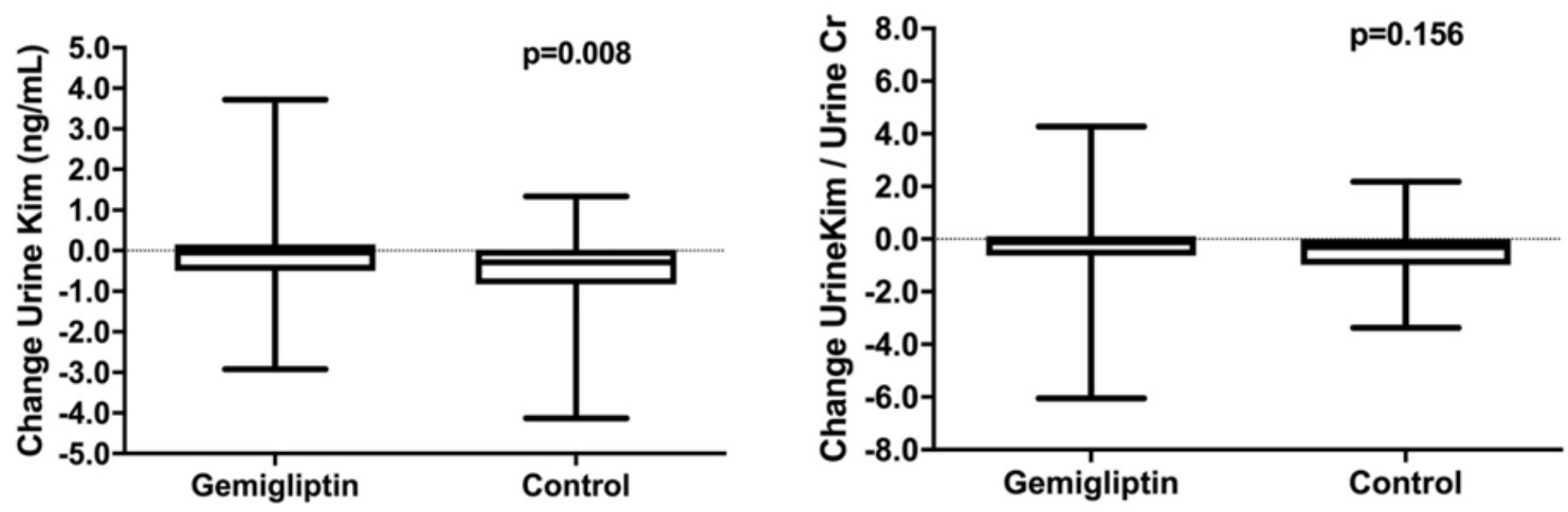

Figure 7

Comparisons between urine KIM-1 and urine KIM-1 factor for urine creatinine in both groups at baseline and at the end of study

\section{Supplementary Files}

This is a list of supplementary files associated with this preprint. Click to download.

- SupplementalFileDPP4.docx 